\title{
A study of Mental Health Status in Healthcare Workers posted in a dedicated COVID-19 hospital
}

\author{
Minakshi Parikh ${ }^{1}$, Srinivasa Kartik Valipay ${ }^{2}$, Rajesh $\mathrm{Kumar}^{3}$, Aana $\mathrm{Shah}^{4}$, Parth Kansagra ${ }^{5}$, \\ Khushboo Mathuria ${ }^{6}$, Vrunda Patel ${ }^{7}$, Aatman Parikh ${ }^{8}$ \\ ${ }^{1}$ Professor and Head, Department of Psychiatry, B.J. Medical College \& Civil Hospital, Ahmedabad. \\ ${ }^{2}$ Ex-Resident Doctor, Department of Psychiatry, B.J. Medical College \& Civil Hospital, Ahmedabad. \\ ${ }^{3}$ Additional Professor, Department of Psychiatry, B.J. Medical College \& Civil Hospital, Ahmedabad. \\ ${ }^{4}$ Ex-Resident Doctor, Department of Psychiatry, B.J. Medical College \& Civil Hospital, Ahmedabad. \\ ${ }^{5,6}$ Resident Doctor, Department of Psychiatry, B.J. Medical College \& Civil Hospital, Ahmedabad. \\ ${ }^{7}$ Ex-Resident Doctor, Department of Psychiatry, B.J. Medical College \& Civil Hospital, Ahmedabad. \\ ${ }^{8}$ Resident Doctor, Department of Psychiatry, G.C.S. Medical College, Ahmedabad. \\ Corresponding author: Minakshi Parikh \\ Email -drmnparikhpp@gmail.com
}

\begin{abstract}
Background: The massive toll due to COVID-19 has resulted in stretching of resources of healthcare systems and has led to unique demands on healthcare workers (HCWs) that are not seen in usual practice, which predispose them to mental health problems. The aim of the study was to assess mental health status in healthcare workers posted in COVID hospital as Mental health issues may impact decision making among HCWs, leading to medical errors and may also impact wellbeing in the long term.

Methodology: This was a cross-sectional study. 799 HCWs posted in the COVID-19 wing of a tertiary care hospital were studied. Socio-demographic details were recorded and a Gujarati version of GHQ-28 was applied. A total score of $\geq 4$ on GHQ-28 was considered indicative of 'caseness' or 'psychiatric morbidity'. Association of outcomes like total GHQ-28 score, and responses on individual items of GHQ indicative of fatigue, sleep disturbances, stress, irritability, anxiety and others with variables like age, gender, profession, years of experience, marital status and family type was assessed.

Results: 40 individuals (5\%) reported at least one of the symptoms. The most commonly reported symptom was sleep disturbance, reported by 38 participants $(4.75 \%)$, followed by stress in 34 participants (4.25\%), fatigue in 29 participants (3.62\%) and anxiety in 24 (3.00\%). Decision-making problems, hopelessness, death wishes/suicidal ideation were rare.

Conclusion: Age $>45$ years was a significant predictor for GHQ-28 total score being $\geq 4$ and stress, experience $>5$ years was statistically significantly associated with sleep disturbance, and being female was a significant predictor for fatigue.
\end{abstract}

Keywords: COVID-19, Mental Health, Stress, Sleep Disturbances, Anxiety, Fatigue

(Paper received $-9^{\text {th }}$ February 2021, Peer review completed $-5^{\text {th }}$ April 2021)

(Accepted $-8^{\text {th }}$ June 2021, Published $-5^{\text {th }}$ January 2022)

\section{INTRODUCTION}

The novel coronavirus disease (COVID-19) was first identified in Wuhan City, Hubei Province, China. The rapid spread of this disease has resulted in the World Health Organization (WHO) declaring it a pandemic - a public health emergency of international concern - on 11th March 2020 [1]. The global toll is massive, with the disease affecting 18.14 million people worldwide and resulting in 6,91,013 deaths as of 15th June 2020 [2]. The pandemic has gripped India as well, with 22,15,074 cases as of 15 th June 2020 and a total of 
44,386 deaths [2]. One of the worst affected states accounting for a large majority of these cases is Gujarat [3]. Healthcare workers (HCWs) have been on the frontline in this pandemic, working with a dailyincreasing caseload at increased risk to their physical health. Anxiety, stress, poor sleep quality are factors commonly seen during infectious disease epidemics among healthcare staff, with depression and posttraumatic stress disorder as a fall-out when these epidemics have eventually trailed off [4-5]. Prolonged quarantine periods for health workers as well as potential social discrimination among unquarantined workers have already been addressed [6-7]. This may create quite an emotional impact on HCWs.

Mental health issues may impact decision making among HCWs and may lead to medical errors. Acute stress in the short-term may impact wellbeing in the long term as well. At the time of our literature review, there have been few studies on the psychological impact of infectious diseases epidemics on the mental health of healthcare workers in India, and especially in Gujarat. We hope to partly address this existing gap in this literature with our study.

\section{METHODOLOGY}

The study was approved by an institutional ethics committee. The study was a single-center, cross-sectional survey, covering $799 \mathrm{HCWs}$ including doctors, nurses and other support workers working in the COVID wing of a tertiary care hospital in Ahmedabad between 21/4/2020 and 4/08/2020. All consenting healthcare workers posted in the COVID wing during the aforementioned period were included. Those unwilling to provide consent were excluded from the study. The subjects were communicated with using video calls or telephonically, as appropriate. Socio-demographic details were recorded using a semistructured proforma, and a Gujarati version of General Health Questionnaire-28 (GHQ-28) was applied.

\section{Instruments}

- A semi-structured proforma which was used for recording sociodemographic data, comprising information about age, sex, years of experience, marital status, and family type (joint or nuclear) and experience in their field of work in years

- General Health Questionnaire-28 (GHQ-28): Developed by Goldberg in 1978[8]. It consists of 28 questions in which Questions 1-7 (subscale A) evaluate somatic symptoms, Questions 8-14 (subscale B) evaluate anxiety/insomnia, Questions 15-21 (subscale C) evaluate social dysfunction and Questions 22 to 28 (subscale D) evaluate depression. For each item, 4 possible answers are available (0-not at all, 0-no more than usual, 1-rather more than usual, 1-much more than usual). A cut-off of 4 or more was considered indicative of psychiatric morbidity or 'caseness' [9]. Validity and reliability of the scale have been shown to be satisfactory. Test-retest and inter and intra-rater reliability have been found to be high [10-11]. The scale has been translated into and validated in Gujarati language. Apart from the total score, we considered responses of 'rather more than usual' or 'much more than usual' on specific questions on the questionnaire to be indicative of certain symptoms. The following questions of the GHQ-28 were used for this purpose A3 - Fatigue, B1\& B2 - Sleep problems, B3 - Stress, B4 - Irritability, B5 \& 7 - Anxiety, C6 - decision making, D2 Hopelessness, D3-D7: Death wishes/suicidal ideation.

The data were tabulated using Microsoft Excel. Outcomes including the category of total GHQ-28 score (< 4 or $\geq 4$ ) and a response of ' 1 ' (rather more than usual or much more than usual) on any of the above mentioned GHQ-28 questions were considered dependent variables and their association with variables like age, gender, profession, years of experience, marital status and family type were assessed.

\section{STATISTICAL ANALYSIS}

Chi square test and independent sample t-test were used to assess associations and Multivariate regression analysis was used to test the strength of associations to assess for potential risk factors for the aforementioned outcomes, and the results are displayed as Odds ratios (ORs) with 95\% confidence interval (CI) and p-values, after controlling for confounders. Post data collection, psychological assistance was offered to all participants 
and provided to those who sought the same. Information of a psychological assistance helpline was also provided to all participants in case they needed assistance in the future.

\section{RESULTS}

\section{Sample Characteristics}

Our sample comprised of 799 healthcare workers belonging to various departments in our hospital. A majority of the individuals surveyed were female (68.59\%), nursing staff (47.30\%), married (57.07\%), living in nuclear families $(51.94 \%)$, between 18 -30 years of age $(61.83 \%)$ and had $<5$ years of experience in their roles $(53.20 \%)$. Of 799 individuals, 40 were found to have a GHQ-28 total score $\geq 4$, which we considered indicative of "caseness" or "psychiatric morbidity". The most commonly reported symptom was sleep disturbance, reported by 38 participants (4.75\%), followed by stress in 34 participants (4.25\%), fatigue in 29 participants (3.62\%) and anxiety in $24(3.00 \%)$. Association of each symptom with variables like age, gender, profession, years of experience, marital status, and family type was assessed using multivariate logistical regression analysis. After controlling for confounders, Age $>45$ years was found to be statistically significantly associated with a GHQ-28 total score $\geq 4$ (OR 5.11, p 0.044) (table 2); Work experience between $5-10$ years (OR 7.32, p 0.013) and $>10$ years (OR 7.09, p 0.036) was found to be associated with sleep disturbance (Table 3). Support workers were least affected by sleep disturbance when compared to other groups and this difference was statistically significant (OR 0.08, p 0.026) (table 3); Age $>45$ years was also statistically significantly associated with stress (OR: 7.17, p 0.028) (table 4) and individuals in this age group also reported anxiety more frequently as compared to other groups, but this association was not statistically significant (table 5); Fatigue was more commonly experienced by females and this difference was statistically significant. Other symptoms like irritability, problems in decision making, hopelessness and death wishes/suicidal ideation were infrequently reported and were not statistically significantly associated with any of the variables considered.

\section{DISCUSSION}

In our study, out of 799 respondents, we had 40 individuals (5.0\%) with a GHQ-28 total score $\geq 4$, which we considered indicative of "caseness". This is in contrast to previous studies which reported a high prevalence of psychological problems [12-14]. Training of HCWs in specific aspects related to the care of COVID patients, and also in recognising and dealing with psychological problems in themselves before being posted in the COVID wing, adequate staffing, ensuring adequate breaks (the number of days of duty was equal to the number of quarantine days provided throughout the duration of this study), a majority of the participants being locals and having available social supports are factors which have possibly contributed to a low number of symptomatic individuals in our setting. After controlling for confounders, age $>45$ years was found to be statistically significantly associated with GHQ-28 total score of $\geq 4$. Higher responsibility related to patient care, presence of comorbidities, worry about own safety \& infecting family, maybe some of the factors that could explain this association.

In our study, the most commonly reported symptom was sleep disturbance, reported by 38 respondents $(4.75 \%)$. After controlling for confounders, duration of experience $>5$ years was found to be strongly associated with sleep disturbance (OR 7.32, p 0.013 for individuals between 5-10 years of experience and OR 7.09, p 0.036 for individuals with $>10$ years of experience). Also, of the listed professions, support workers reported sleep disturbance the least, with the difference between groups being statistically significant (OR 0.08, p 0.026). This could be explained by a role difference, where nurses and residents/interns have more responsibility towards clinical care than support workers. This difference could also probably be explained by the amount of direct contact with infected inpatients, with nurses and residents/interns having the most amount of direct contact and involvement with infected patients, with support workers having lesser direct/less prolonged contact.

The next most commonly reported symptom was stress, reported by $34(4.25 \%)$ respondents. After controlling for confounders, age $>45$ years was found to be statistically significantly associated with stress (OR: 7.17, p 0.028). This finding is in tune with a recent study which noted that factors like concern about 
infecting families, worry about own safety, exhaustion due to prolonged use of personal protective equipment and at times perceived inadequacy of personal protective equipment are contributors to increased stress in individuals aged 31-50 and older [15].

Table 1: Sample Characteristics ( $=799)$

\begin{tabular}{|c|c|c|}
\hline \multirow{4}{*}{ Age } & $18-30$ & $494(61.83 \%)$ \\
\cline { 2 - 3 } & $30-45$ & $232(29.03 \%)$ \\
\cline { 2 - 3 } & $>45$ & $73(9.14 \%)$ \\
\hline \multirow{3}{*}{ Gender } & Male & $251(31.41 \%)$ \\
\cline { 2 - 3 } & Female & $548(68.59 \%)$ \\
\hline \multirow{3}{*}{ Profession } & Resident & $163(20.4 \%)$ \\
\cline { 2 - 3 } & Intern & $145(18.14 \%)$ \\
\cline { 2 - 3 } & Nursing Staff & $378(47.30 \%)$ \\
\cline { 2 - 3 } & Other support workers & $113(14.14 \%)$ \\
\hline \multirow{3}{*}{ Experience (in years) } & $<5$ years & $425(53.20 \%)$ \\
\cline { 2 - 3 } & 5 to 10 years & $184(23.02 \%)$ \\
\cline { 2 - 3 } & $>10$ years & $190(23.78 \%)$ \\
\hline \multirow{2}{*}{ Marital Status } & Unmarried & $343(42.93 \%)$ \\
\cline { 2 - 3 } & Married & $456(57.07 \%)$ \\
\hline Family Type & Nuclear & $415(51.94 \%)$ \\
\cline { 2 - 3 } & Joint & $384(48.06 \%)$ \\
\hline
\end{tabular}

Table 2: Association of Variables with GHQ-28 total score category

\begin{tabular}{|c|c|c|c|c|c|}
\hline \multicolumn{2}{|c|}{ Independent Variable } & $<4$ & $\geq 4$ & OR (univariable) & OR (multivariable) \\
\hline \multirow[t]{3}{*}{ Age } & 18-30 Years & $476(96.4)$ & $18(3.6)$ & - & - \\
\hline & $31-45$ Years & $218(94.0)$ & $14(6.0)$ & $\begin{array}{c}1.70 \\
(0.83-3.48, p=0.147)\end{array}$ & $\begin{array}{c}2.37 \\
(0.67-8.37, p=0.180)\end{array}$ \\
\hline & $>45$ Years & $65(89.0)$ & $8(11.0)$ & $\begin{array}{c}3.25 \\
(1.36-7.79, \mathrm{p}=0.008)\end{array}$ & $\begin{array}{c}5.11 \\
(1.04-25.10, p=0.044)\end{array}$ \\
\hline \multirow[t]{2}{*}{ Gender } & Male & $243(96.8)$ & $8(3.2)$ & - & - \\
\hline & Female & $516(94.2)$ & $32(5.8)$ & $\begin{array}{c}1.88 \\
(0.86-4.15, \mathrm{p}=0.116)\end{array}$ & $\begin{array}{c}1.69 \\
(0.67-4.27, \mathrm{p}=0.266)\end{array}$ \\
\hline \multirow[t]{4}{*}{ Profession } & Resident & $157(96.3)$ & $6(3.7)$ & - & - \\
\hline & Intern & $139(95.9)$ & $6(4.1)$ & $\begin{array}{c}1.13 \\
(0.36-3.58, \mathrm{p}=0.836)\end{array}$ & $\begin{array}{c}1.36 \\
(0.42-4.40, \mathrm{p}=0.609)\end{array}$ \\
\hline & Nursing staff & $355(93.9)$ & $23(6.1)$ & $\begin{array}{c}1.70 \\
(0.68-4.25, \mathrm{p}=0.260)\end{array}$ & $\begin{array}{c}0.98 \\
(0.20-4.72, \mathrm{p}=0.984)\end{array}$ \\
\hline & $\begin{array}{l}\text { Support } \\
\text { worker }\end{array}$ & $108(95.6)$ & $5(4.4)$ & $\begin{array}{c}1.21 \\
(0.36-4.07, \mathrm{p}=0.756)\end{array}$ & $\begin{array}{c}0.66 \\
(0.10-4.36, \mathrm{p}=0.666)\end{array}$ \\
\hline \multirow{3}{*}{$\begin{array}{c}\text { Years of } \\
\text { experience }\end{array}$} & $<5$ Years & $411(96.7)$ & $14(3.3)$ & - & - \\
\hline & 5-10 Years & $172(93.5)$ & $12(6.5)$ & $\begin{array}{c}2.05 \\
(0.93-4.52, \mathrm{p}=0.076)\end{array}$ & $\begin{array}{c}3.07 \\
(0.79-11.99, p=0.107)\end{array}$ \\
\hline & $>10$ Years & $176(92.6)$ & $14(7.4)$ & $\begin{array}{c}2.34 \\
(1.09-5.00, \mathrm{p}=0.029)\end{array}$ & $\begin{array}{c}1.73 \\
(0.32-9.35, \mathrm{p}=0.522)\end{array}$ \\
\hline \multirow[t]{2}{*}{ Marital status } & Married & $430(94.3)$ & $26(5.7)$ & - & - \\
\hline & $\begin{array}{c}\text { Unmarried/ } \\
\text { Widowed }\end{array}$ & $329(95.9)$ & $14(4.1)$ & $\begin{array}{c}0.70 \\
(0.36-1.37, \mathrm{p}=0.301)\end{array}$ & $\begin{array}{c}2.58 \\
(0.53-12.55, \mathrm{p}=0.240)\end{array}$ \\
\hline \multirow[t]{2}{*}{ Family type } & Nuclear & $393(94.7)$ & $22(5.3)$ & - & - \\
\hline & Joint & $366(95.3)$ & $18(4.7)$ & $\begin{array}{c}0.88 \\
(0.46-1.66, \mathrm{p}=0.691)\end{array}$ & $\begin{array}{c}0.66 \\
(0.32-1.35, \mathrm{p}=0.251)\end{array}$ \\
\hline
\end{tabular}

Abbreviations: $G H Q-28=$ General Health Questionnaire 28 item version, $O R=$ Odds ratio 
Table 3: Association of variables with sleep disturbance as the dependent variable

\begin{tabular}{|c|c|c|c|c|c|}
\hline \multicolumn{2}{|c|}{ Independent Variable } & \multirow{2}{*}{$\begin{array}{c}\text { Absent } \\
479(97.0)\end{array}$} & \multirow{2}{*}{$\frac{\text { Present }}{15(3.0)}$} & \multirow{2}{*}{$\begin{array}{c}\text { OR (univariable) } \\
- \\
\end{array}$} & \multirow{2}{*}{$\frac{\text { OR (multivariable) }}{-}$} \\
\hline Age & 18-30 Years & & & & \\
\hline & 31-45 Years & $216(93.1)$ & $16(6.9)$ & $\begin{array}{c}2.37 \\
(1.15-4.87, \mathrm{p}=0.020)\end{array}$ & $\begin{array}{c}2.02 \\
(0.55-7.38, \mathrm{p}=0.286)\end{array}$ \\
\hline & $>45$ Years & $66(90.4)$ & $7(9.6)$ & $\begin{array}{c}3.39 \\
(1.33-8.61, \mathrm{p}=0.010) \\
\end{array}$ & $\begin{array}{c}2.50 \\
(0.50-12.44, \mathrm{p}=0.263)\end{array}$ \\
\hline \multirow[t]{2}{*}{ Gender } & Male & $245(97.6)$ & $6(2.4)$ & - & - \\
\hline & Female & $516(94.2)$ & $32(5.8)$ & $\begin{array}{c}2.53 \\
(1.05-6.14, \mathrm{p}=0.040)\end{array}$ & $\begin{array}{c}2.11 \\
(0.75-5.92, p=0.157)\end{array}$ \\
\hline \multirow[t]{4}{*}{ Profession } & Resident & $156(95.7)$ & $7(4.3)$ & - & - \\
\hline & Intern & $141(97.2)$ & $4(2.8)$ & $\begin{array}{c}0.63 \\
(0.18-2.21, \mathrm{p}=0.472)\end{array}$ & $\begin{array}{c}0.91 \\
(0.25-3.39, \mathrm{p}=0.891)\end{array}$ \\
\hline & $\begin{array}{l}\text { Nursing } \\
\text { staff }\end{array}$ & $354(93.7)$ & $24(6.3)$ & $\begin{array}{c}1.51 \\
(0.64-3.58, p=0.348)\end{array}$ & $\begin{array}{c}0.19 \\
(0.03-1.28, \mathrm{p}=0.088)\end{array}$ \\
\hline & $\begin{array}{l}\text { Support } \\
\text { worker }\end{array}$ & $110(97.3)$ & $3(2.7)$ & $\begin{array}{c}0.61 \\
(0.15-2.40, \mathrm{p}=0.478)\end{array}$ & $\begin{array}{c}0.08 \\
(0.01-0.73, \mathrm{p}=0.026)\end{array}$ \\
\hline \multirow{3}{*}{$\begin{array}{l}\text { Years of } \\
\text { experience }\end{array}$} & $<5$ Years & 415 (97.6) & $10(2.4)$ & - & - \\
\hline & 5-10 Years & $173(94.0)$ & $11(6.0)$ & $\begin{array}{c}2.64 \\
(1.10-6.33, p=0.030)\end{array}$ & $\begin{array}{c}7.32 \\
(1.53-35.14, p=0.013)\end{array}$ \\
\hline & $>10$ Years & $173(91.1)$ & $17(8.9)$ & $\begin{array}{c}4.08 \\
(1.83-9.08, p=0.001)\end{array}$ & $\begin{array}{c}7.09 \\
(1.13-44.32, p=0.036)\end{array}$ \\
\hline \multirow[t]{2}{*}{ Marital status } & Married & $429(94.1)$ & $27(5.9)$ & - & - \\
\hline & $\begin{array}{l}\text { Unmarried/ } \\
\text { Widowed }\end{array}$ & $332(96.8)$ & $11(3.2)$ & $\begin{array}{c}0.53 \\
(0.26-1.08, \mathrm{p}=0.079)\end{array}$ & $\begin{array}{c}1.32 \\
(0.20-8.57, \mathrm{p}=0.774)\end{array}$ \\
\hline \multirow[t]{2}{*}{ Family type } & Nuclear & $396(95.4)$ & $19(4.6)$ & - & - \\
\hline & Joint & $365(95.1)$ & $19(4.9)$ & $\begin{array}{c}1.08 \\
(0.57-2.08, \mathrm{p}=0.806)\end{array}$ & $\begin{array}{c}0.93 \\
(0.45-1.92, \mathrm{p}=0.842)\end{array}$ \\
\hline
\end{tabular}

Table 4: Association of variables with stress as the dependent variable

\begin{tabular}{|c|c|c|c|c|c|}
\hline \multicolumn{2}{|c|}{ Independent variable } & \multirow{2}{*}{$\begin{array}{r}\text { Absent } \\
480(97.2)\end{array}$} & \multirow{2}{*}{$\begin{array}{l}\text { Present } \\
14(2.8)\end{array}$} & \multirow[t]{2}{*}{ OR (univariable) } & \multirow[t]{2}{*}{ OR (multivariable) } \\
\hline Age & 18-30 Years & & & & \\
\hline & 31-45 Years & $220(94.8)$ & $12(5.2)$ & $\begin{array}{r}1.87 \\
(0.85-4.11, p=0.119)\end{array}$ & $\begin{array}{r}3.10 \\
(0.75-12.82, p=0.119)\end{array}$ \\
\hline & $>45$ Years & $65(89.0)$ & $8(11.0)$ & $(1.70-10.45, p=0.002)$ & $\begin{array}{r}7.17 \\
(1.24-41.38, p=0.028)\end{array}$ \\
\hline \multirow[t]{2}{*}{ Gender } & Male & $244(97.2)$ & $7(2.8)$ & - & - \\
\hline & Female & $521(95.1)$ & $27(4.9)$ & $\begin{array}{r}1.81 \\
(0.78-4.21, p=0.170)\end{array}$ & $\begin{array}{r}1.50 \\
(0.55-4.10, p=0.431)\end{array}$ \\
\hline \multirow[t]{4}{*}{ Profession } & Resident & $158(96.9)$ & $5(3.1)$ & - & - \\
\hline & Intern & $140(96.6)$ & $5(3.4)$ & $\begin{array}{r}1.13 \\
(0.32-3.98, p=0.851)\end{array}$ & $\begin{array}{r}1.34 \\
(0.37-4.83, p=0.659)\end{array}$ \\
\hline & Nursing staff & $356(94.2)$ & $22(5.8)$ & $\begin{array}{r}1.95 \\
(0.73-5.25, \mathrm{p}=0.185) \\
\end{array}$ & $\begin{array}{r}0.68 \\
(0.11-4.34, \mathrm{p}=0.687) \\
\end{array}$ \\
\hline & $\begin{array}{l}\text { Support } \\
\text { worker }\end{array}$ & $111(98.2)$ & $2(1.8)$ & $\begin{array}{r}0.57 \\
(0.11-2.99, p=0.505)\end{array}$ & $\begin{array}{r}0.17 \\
(0.02-1.84, \mathrm{p}=0.144)\end{array}$ \\
\hline \multirow{3}{*}{$\begin{array}{l}\text { Years of } \\
\text { experience }\end{array}$} & $<5$ Years & $414(97.4)$ & $11(2.6)$ & - & - \\
\hline & 5-10 Years & $175(95.1)$ & $9(4.9)$ & $\begin{array}{r}1.94 \\
(0.79-4.75, p=0.150)\end{array}$ & $\begin{array}{r}3.03 \\
(0.60-15.21, p=0.178)\end{array}$ \\
\hline & $>10$ Years & $176(92.6)$ & $14(7.4)$ & $\begin{array}{r}2.99 \\
(1.33-6.72, p=0.008)\end{array}$ & $\begin{array}{r}1.76 \\
(0.26-12.03, p=0.562)\end{array}$ \\
\hline
\end{tabular}




\begin{tabular}{|c|c|c|c|c|c|}
\hline \multirow[t]{2}{*}{ Marital status } & Married & $433(95.0)$ & $23(5.0)$ & - & - \\
\hline & $\begin{array}{l}\text { Unmarried/ } \\
\text { Widowed }\end{array}$ & $332(96.8)$ & $11(3.2)$ & $\begin{array}{r}0.62 \\
(0.30-1.30, p=0.207)\end{array}$ & $\begin{array}{r}1.93 \\
(0.30-12.43, p=0.488)\end{array}$ \\
\hline \multirow[t]{2}{*}{ Family type } & Nuclear & 397 (95.7) & $18(4.3)$ & - & 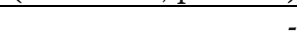 \\
\hline & Joint & $368(95.8)$ & $16(4.2)$ & $\begin{array}{r}0.96 \\
(0.48-1.91, p=0.905)\end{array}$ & $\begin{array}{r}0.79 \\
(0.37-1.68, \mathrm{p}=0.536)\end{array}$ \\
\hline
\end{tabular}

Table 5: Association of variables with anxiety as the dependent variable

\begin{tabular}{|c|c|c|c|c|c|}
\hline \multicolumn{2}{|c|}{ Independent variable } & Absent & Present & OR (univariable) & OR (multivariable) \\
\hline \multirow[t]{3}{*}{ Age } & $18-30$ years & $482(97.6)$ & $12(2.4)$ & - & - \\
\hline & $31-45$ years & $225(97.0)$ & $7(3.0)$ & $\begin{array}{r}1.25 \\
(0.49-3.22, \mathrm{p}=0.644)\end{array}$ & $\begin{array}{r}1.69 \\
(0.42-6.84, p=0.461)\end{array}$ \\
\hline & $>45$ years & $68(93.2)$ & $5(6.8)$ & $\begin{array}{r}2.95 \\
(1.01-8.64, \mathrm{p}=0.048)\end{array}$ & $\begin{array}{r}6.40 \\
(0.87-47.26, p=0.069)\end{array}$ \\
\hline \multirow[t]{2}{*}{ Gender } & Male & $246(98.0)$ & $5(2.0)$ & - & - \\
\hline & Female & $529(96.5)$ & $19(3.5)$ & $\begin{array}{r}1.77 \\
(0.65-4.79, p=0.263)\end{array}$ & $\begin{array}{r}1.67 \\
(0.52-5.39, p=0.393)\end{array}$ \\
\hline \multirow[t]{4}{*}{ Profession } & Resident & $160(98.2)$ & $3(1.8)$ & - & \\
\hline & Intern & $141(97.2)$ & $4(2.8)$ & $\begin{array}{r}1.51 \\
(0.33-6.88, p=0.592)\end{array}$ & $\begin{array}{r}1.84 \\
(0.39-8.62, p=0.438)\end{array}$ \\
\hline & $\begin{array}{l}\text { Nursing } \\
\text { staff }\end{array}$ & $363(96.0)$ & $15(4.0)$ & $\begin{array}{r}2.20 \\
(0.63-7.72, p=0.217)\end{array}$ & $\begin{array}{r}0.61 \\
(0.07-5.70, p=0.666)\end{array}$ \\
\hline & $\begin{array}{l}\text { Support } \\
\text { worker }\end{array}$ & $111(98.2)$ & $2(1.8)$ & $\begin{array}{r}0.96 \\
(0.16-5.84, \mathrm{p}=0.966)\end{array}$ & $\begin{array}{r}0.20 \\
(0.01-3.03, p=0.246)\end{array}$ \\
\hline \multirow{3}{*}{$\begin{array}{l}\text { Years of } \\
\text { experience }\end{array}$} & $<5$ Years & $416(97.9)$ & $9(2.1)$ & - & - \\
\hline & 5-10 Years & $176(95.7)$ & $8(4.3)$ & $\begin{array}{r}2.10 \\
(0.80-5.53, \mathrm{p}=0.133)\end{array}$ & $\begin{array}{r}1.67 \\
(0.36-7.76, p=0.512)\end{array}$ \\
\hline & $>10$ Years & $183(96.3)$ & $7(3.7)$ & $\begin{array}{r}1.77 \\
(0.65-4.82, p=0.265) \\
\end{array}$ & $\begin{array}{r}0.48 \\
(0.06-3.92, p=0.491) \\
\end{array}$ \\
\hline \multirow{2}{*}{$\begin{array}{l}\text { Marital } \\
\text { status }\end{array}$} & Married & $439(96.3)$ & $17(3.7)$ & - & \\
\hline & $\begin{array}{l}\text { Unmarried } \\
\text { /Widowed }\end{array}$ & $336(98.0)$ & $7(2.0)$ & $\begin{array}{r}0.54 \\
(0.22-1.31, \mathrm{p}=0.173) \\
\end{array}$ & $\begin{array}{r}0.42 \\
(0.05-3.34, \mathrm{p}=0.415)\end{array}$ \\
\hline \multirow[t]{2}{*}{ Family type } & Nuclear & 402 (96.9) & $13(3.1)$ & - & \\
\hline & Joint & $373(97.1)$ & $11(2.9)$ & $\begin{array}{r}0.91 \\
(0.40-2.06, \mathrm{p}=0.825)\end{array}$ & $\begin{array}{r}0.75 \\
(0.31-1.82, p=0.521)\end{array}$ \\
\hline
\end{tabular}

(Abbreviations: GHQ-28 - General Health Questionnaire 28 item version; OR - Odds ratio)

While conventional wisdom would probably predict more experience to be protective, it could be said that COVID as a novel disease was a new predicament for both the experienced and relatively inexperienced workers alike, and this could be a factor in explaining the findings in individuals with age $>45$ and more years of experience, along with the aforementioned factors.

Fatigue was reported by 29 participants (3.63\%). After controlling for confounders, female gender was found to be statistically significantly associated with fatigue (OR $4.20, \mathrm{p} 0.032$ ). Shaukat et al. in a recent review of 10 articles discussed that frontline female nurses experienced increased levels of fatigue [16]. Another recent study assessing the social stigma during COVID-19 and impact on HCWs also found higher levels of burnout and fatigue in female workers [17]. Conversely, a study by Zhang et al found more feelings of fatigue in male HCWs as compared to females, although the difference was not statistically significant. Long hours in PPEs, Infection with COVID-19, menstrual periods during duties are some of the common factors that 
could explain the above findings. The high prevalence of anaemia in our region, and also the extremely hot climate in this region during the period of this study, may also have been contributing factors.

Anxiety was reported by 24 participants (3.0\%). We found that anxiety was reported more frequently by individuals $>45$ years of age, but the association was not statistically significant (OR 6.41, p 0.06). This finding is somewhat similar to Liang $\mathrm{Y}$ et al. who found that there was no significant difference in self-rated anxiety and depression scores among staff in the COVID-19 associated department and other departments [18]. Several recent studies though have found increased anxiety in healthcare workers [16]. In various studies, the inadequacy of PPE, worry about transmitting the infection to family, worry about own health were sources of anxiety, while the availability of social supports was found to be protective against anxiety [13-15]. In our setting, we had a high number of HCWs who were already doing their second or third stints in the COVID hospital, and many of the HCWs were also local, with social supports available. PPE inadequacy was also not a concern in our setting. These factors, along with the ones mentioned earlier like adequate staffing, adequate gaps between COVID wing postings, training in relevant aspects immediately before postings, could have contributed to the low number of participants reporting anxiety symptoms.

The other symptoms like irritability, hopelessness, death wishes/suicidal ideation were reported by very few participants. This is consistent with some previous research about psychological symptoms in HCWs during epidemics, wherein depressive symptoms or symptoms of PTSD were more of chronic sequelae (and stress, anxiety and sleep problems were seen earlier) while our sample belonged to an early part of the pandemic response.

\section{Limitations}

- The scope of the study was limited as the sample was entirely from one institution, and inclusion of subjects from more institutions could provide a better estimate of generalisable findings

- This was a screening study, and diagnostic thresholds were not applied

- The severity of symptoms was not measured in the study. Also, since this was a cross-sectional study, any change in the status (presence/absence)/severity of symptoms in the subjects over time could not be tracked

\section{CONCLUSIONS}

The overall frequency of psychological symptoms in our study population was quite low, as compared to other recent studies on the same topic. Age $>45$ years was a significant predictor for GHQ-28 total score being $\geq 4$ and stress, experience $>5$ years was statistically significantly associated with sleep disturbance, and being female was a significant predictor for fatigue, in healthcare workers involved in the management of COVID patients.

\section{REFERENCES}

1. WHO Director-General's opening remarks at the media briefing on COVID-19 - 11 March 2020. World Health Organization. 2020b. https://www.who.int/dg/speeches/detail/who-director-general-s-opening-remarks-atthe-media-briefing-on-covid-19---11-march-2020

2. WHO Coronavirus Disease (COVID-19) Dashboard. World Health Organization. 2020a. https://covid19.who.int/

3. COVID-19: Gujarat records 1,009 cases; 22 die, 974 recover. Outlook. 2020. https://www.outlookindia.com/newsscroll/covid19-gujarat-records-1009-cases-22-die-974-recover/1908416

4. Tan BY, Chew NW, Lee GK, Jing M, Goh Y, Yeo LL, Zhang K, Chin HK, Ahmad A, Khan FA, Shanmugam GN. Psychological impact of the COVID-19 pandemic on health care workers in Singapore. Ann Intern Med 2020;173(4):317-20.

5. Dutheil F, Mondillon L, Navel V. PTSD as the second tsunami of the SARS-Cov-2 pandemic. Psychol Med 2020;24:1-2.

6. Singh R, Subedi M. COVID-19 and stigma: Social discrimination towards frontline healthcare providers and COVID-19 recovered patients in Nepal. Asian J Psychiatry 2020;102222

7. Bagcchi S. Stigma during the COVID-19 pandemic. Lancet Infect Dis 2020;20(7):782.

8. Goldberg D. Manual of the general health questionnaire. Nfer Nelson; 1978.

9. Jackson C. The general health questionnaire. Occup Med (Lond) 2007;57(1):79-86. 
10. Robinson RG, Price TR. Post-stroke depressive disorders: a follow-up study of 103 patients. Stroke 1982;13(5):635-41.

11. Failde I, Ramos I, Fernandez-Palacin F. Comparison between the GHQ-28 and SF-36 (MH 1-5) for the assessment of the mental health in patients with ischaemic heart disease. Eur J Epidemiol 2000;16(4):311-6.

12. Chatterjee P, Anand T, Singh KJ, Rasaily R, Singh R, Das S, Singh H, Praharaj I, Gangakhedkar RR, Bhargava B, Panda S. Healthcare workers \& SARS-CoV-2 infection in India: A case-control investigation in the time of COVID-19. Indian J Med Res 2020;151(5):459-65.

13. Lai J, Ma S, Wang Y, Cai Z, Hu J, Wei N, Wu J, Du H, Chen T, Li R, Tan H. Factors associated with mental health outcomes among health care workers exposed to coronavirus disease 2019. JAMA New Open 2020;3(3):e203976.

14. Rossi R, Socci V, Pacitti F, Di Lorenzo G, Di Marco A, Siracusano A, Rossi A. Mental health outcomes among frontline and second-line health care workers during the coronavirus disease 2019 (COVID-19) pandemic in Italy. JAMA Netw Open 2020;3(5):e2010185.

15. Cai H, Tu B, Ma J, Chen L, Fu L, Jiang Y, Zhuang Q. Psychological impact and coping strategies of frontline medical staff in Hunan between January and March 2020 during the outbreak of coronavirus disease 2019 (COVID-19) in Hubei, China. Med Sci Monit 2020;26:e924171-6.

16. Shaukat N, Ali DM, Razzak J. Physical and mental health impacts of COVID-19 on healthcare workers: A scoping review. Int J Emerg Med 2020;13(1):1-8.

17. Ramaci T, Barattucci M, Ledda C, Rapisarda V. Social stigma during COVID-19 and its impact on HCWs outcomes. Sustainability 2020;12(9):3834-9.

18. Liang Y, Chen M, Zheng X, Liu J. Screening for Chinese medical staff mental health by SDS and SAS during the outbreak of COVID-19. J Psychosom Res 2020;133:110102.

$* * * * * * * * * * * * * * * * * * * * * * * * * * * * * * * * * * * *$

Acknowledgements - Nil

Conflict of Interest - Nil

Funding - Nil 